\title{
On the Maximum Observed Wind Speed in a Randomly Sampled Hurricane
}

\author{
ANDREW R. SOLOW \\ Woods Hole Oceanographic Institution, Woods Hole, Massachusetts
}

(Manuscript received 25 August 2008, in final form 23 September 2009)

\begin{abstract}
There is considerable interest in detecting a long-term trend in hurricane intensity possibly related to largescale ocean warming. This effort is complicated by the paucity of wind speed measurements for hurricanes occurring in the early part of the observational record. Here, results are presented regarding the maximum observed wind speed in a sparsely randomly sampled hurricane based on a model of the evolution of wind speed over the lifetime of a hurricane.
\end{abstract}

\section{Introduction}

There is considerable interest in detecting and understanding historical variations in the intensity of hurricanes as measured, for example, by maximum wind speed. This interest stems in part from a possible connection between hurricane intensity and large-scale climate change (Emanuel 2005). A potentially serious problem in identifying such variation is that only a few chance wind speed measurements may be available for hurricanes in the earliest part of the observational record (Landsea et al. 2004a). The purpose of this note is to describe some general statistical results concerning the maximum observed wind speed in a hurricane that is sparsely observed at random times during its lifetime. These general results are then specialized to a model of the evolution of wind speed within a hurricane based on Emanuel (2000).

\section{The basic result}

In this section, the basic statistical result is outlined. This result is specialized to a particular case in section 3 .

Consider a hurricane with lifetime $(0, T)$. Let $v(t)$ be the maximum wind speed of this hurricane at time $t$. Suppose that this hurricane is observed at random times $t_{1}, t_{2}, \ldots, t_{n}$ during its lifetime and let the random variables

Corresponding author address: Andrew Solow, Woods Hole Oceanographic Institution, Woods Hole, MA 02543.

E-mail: asolow@whoi.edu
$W_{1}, W_{2}, \ldots, W_{n}$ be the maximum wind speeds observed at these times. To begin with, assume that the error in these observations is negligible, so that $W_{j}=v\left(t_{j}\right)$. This assumption is relaxed later.

The distribution function of a randomly observed wind speed $W$ is given by

$$
F(w)=1-\frac{T(w)}{T},
$$

where $T(w)$ is the total time during which wind speed exceeds $w$. The corresponding probability density function (pdf) is given by

$$
f(w)=-\frac{T^{\prime}(w)}{T} .
$$

The support of this pdf-that is, the values of $v$ over which $f(w)$ is positive-has an upper bound at the maximum value $v_{\max }$ of $v(t)$ over the interval $(0, T)$.

Let $W_{(1)}<W_{(2)}<\cdots<W_{(n)}$ be the observed wind speeds ordered from smallest to largest, so that $W_{(n)}$ is the maximum observed wind speed. It is a standard result that the pdf of $W_{(n)}$ is

$$
g(w)=n f(w) F^{n-1}(w)
$$

(David and Nagaraja 2003). Clearly, the support of $g(w)$ also has an upper bound at $v_{\max }$. Beyond that, the behavior of $g(w)$ depends on $F(w)$, which depends in turn on $v(t)$. For large $n$, the distribution of $W_{(n)}$ will converge 
to the Weibull extreme value distribution, which is the only extreme value distribution with finite support. However, the situation of interest here is when $n$ is small.

This basic model can be extended to allow for error in observed wind speed. For example, suppose now that observed wind speed $W_{j}$ is given by the sum

$$
W_{j}=v\left(t_{j}\right)+\varepsilon_{j}
$$

of true wind speed at time $t_{j}$ and a normal observation error with mean 0 and variance $\sigma^{2}$. Under this model, the distribution function of a randomly observed wind speed is given by the convolution

$$
F_{e}(w)=\int_{0}^{\infty} \Phi\left[\frac{(w-v)}{\sigma}\right] f(v) d v
$$

where $\Phi$ is the standard normal distribution function and $f(v)$ is given in (2). Here and below, the subscript $e$ is used to indicate results for the error model in (4). The corresponding pdf is

$$
f_{e}(w)=\frac{1}{\sigma} \int_{0}^{\infty} \varphi\left[\frac{(w-v)}{\sigma}\right] f(v) d v,
$$

where $\varphi$ is the standard normal pdf. Assuming that the errors in the observations are independent, the pdf $g_{e}(w)$ of $W_{(n)}$ has the same form as (3) with $f$ and $F$ replaced by $f_{e}$ and $F_{e}$, respectively. In this case, the support of $W_{(n)}$ is unbounded; therefore, its limiting distribution is no longer Weibull.

\section{A special case}

Emanuel (2000) showed that the evolution of intensity in Atlantic Ocean and western North Pacific Ocean hurricanes whose maximum intensity was not limited by declining potential energy exhibited remarkable regularity, with wind speed increasing linearly by around $12 \mathrm{~m} \mathrm{~s}^{-1}$ day $^{-1}$ to maximum wind speed, followed by a linear decay of around $8 \mathrm{~m} \mathrm{~s}^{-1}$ day $^{-1}$. Based on Emanuel's result, suppose that

$$
\begin{array}{rrrl}
v(t)= & v_{0}+\beta t & 0 \leq t \leq t_{\max } \\
v_{\max }-\gamma\left(t-t_{\max }\right) & t_{\max }<t \leq T
\end{array}
$$

with $\beta$ and $\gamma>0$, where $v_{\max }=v_{0}+\beta t_{\max }$ and $T=$ $t_{\max }+\left(v_{\max }-v_{0}\right) / \gamma$. Under this model, the lifetime of a hurricane begins when wind speed reaches $v_{0}$. Wind speed then increases linearly at rate $\beta$ until reaching a peak of $v_{\max }$ before declining linearly at rate $\gamma$ until it again reaches $v_{0}$ at time $T$.
It is straightforward to show that, in the absence of observation error, a randomly observed wind speed $W$ under this model has a uniform distribution over the interval $\left(v_{0}, v_{\max }\right)$ with distribution function

$$
F(w)=\frac{w-v_{0}}{v_{\max }-v_{0}} \quad v_{0} \leq w \leq v_{\max }
$$

and pdf

$$
f(w)=\frac{1}{v_{\max }-v_{0}} \quad v_{0} \leq w \leq v_{\max }
$$

It follows from (3) that the pdf of $W_{(n)}$ is

$$
g(w)=n \frac{\left(w-v_{0}\right)^{n-1}}{\left(v_{\max }-v_{0}\right)^{n}} .
$$

For $n>1, g(w)$ increases monotonically with $w$, becoming increasingly concave as $n$ increases. The expected value of $W_{(n)}$ is given by

$$
E\left[W_{(n)}\right]=v_{0}+\frac{n}{n+1}\left(v_{\max }-v_{0}\right)<v_{\max } .
$$

The relative underestimation bias in using $W_{(n)}$ as an estimate of $v_{\max }$ is

$$
\frac{\left\{v_{\max }-E\left[W_{(n)}\right]\right\}}{v_{\max }}=\frac{\left(1-v_{0} / v_{\max }\right)}{(n+1)} .
$$

So, for example, if $v_{0} / v_{\max }=0.25$, then the relative underestimation bias is around $38 \%$ for $n=1$ and $13 \%$ for $n=5$.

Turning to the case in which wind speed is observed with normal error, it is straightforward to show that, for the wind speed model in (7), the pdf of a randomly observed wind speed $W$ is given by

$$
f_{e}(w)=\frac{\Phi\left[\left(w-v_{0}\right) / \sigma\right]-\Phi\left[\left(w-v_{\max }\right) / \sigma\right]}{v_{\max }-v_{0}} .
$$

No closed form expressions for $F_{e}(w), g_{e}(w)$, or $E_{e}\left[W_{(n)}\right]$ are available, but it is straightforward to evaluate these numerically. For example, Fig. 1 shows $g_{e}(w)$ for the case $v_{\max }=1, v_{0}=0.25, n=4$, and $\sigma=0.1$. For comparison, Fig. 1 also shows $g(w)$ for the same values of $v_{\max }, v_{0}$, and $n$. In Fig. 2, $E_{e}\left[W_{(n)}\right]$ is plotted against $n$ for these values of $v_{\max }, v_{0}$, and $\sigma$. Again, for comparison, Fig. 2 also shows $E\left[V_{(n)}\right]$ for the same values of $v_{\max }$ and $v_{0}$. It is notable that, by opening the possibility of overestimation, the presence of measurement error actually reduces estimation bias. 


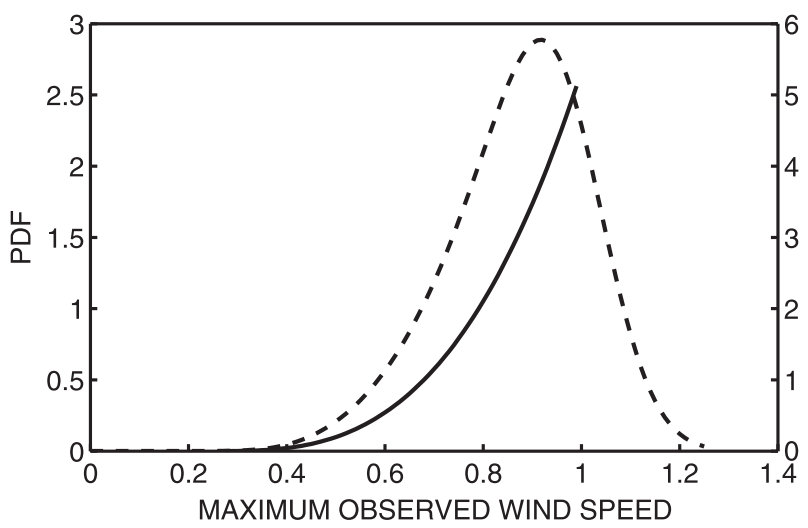

FIG. 1. The pdf of maximum observed wind speed for $v_{\max }=1$, $v_{0}=0.25, n=4$, and $\sigma=0$ (solid) and $\sigma=0.1$ (dashed).

\section{A practical application}

The theoretical results presented in the previous section provide the basis for a rough practical method to correct for underestimation bias. The expression in (11) can be rearranged as

$v_{\max }=\frac{n+1}{n} E\left[W_{(n)}\right]-\frac{1}{n} v_{0} \cong \frac{n+1}{n} E\left[W_{(n)}\right]$.

Thus, a rough correction for underestimation bias can be made by inflating the observed value of $W_{(n)}$ by the factor of $(n+1) / n$, leading to the estimator $\hat{v}_{\max }=$ $W_{(n)}(n+1) / n$. This requires knowledge of only $n$ and $W_{(n)}$.

A simulation experiment based on the reanalyzed National Hurricane Center North Atlantic basin besttrack hurricane database (known as "HURDAT") wind speed measurements for Hurricane Andrew reported in Table 1 of Landsea et al. (2004b) was conducted to assess the performance of $\hat{v}_{\max }$. For this analysis, $v_{0}$ was taken to be $35 \mathrm{kt}\left(1 \mathrm{kt} \approx 0.5 \mathrm{~m} \mathrm{~s}^{-1}\right)$. The data consist of

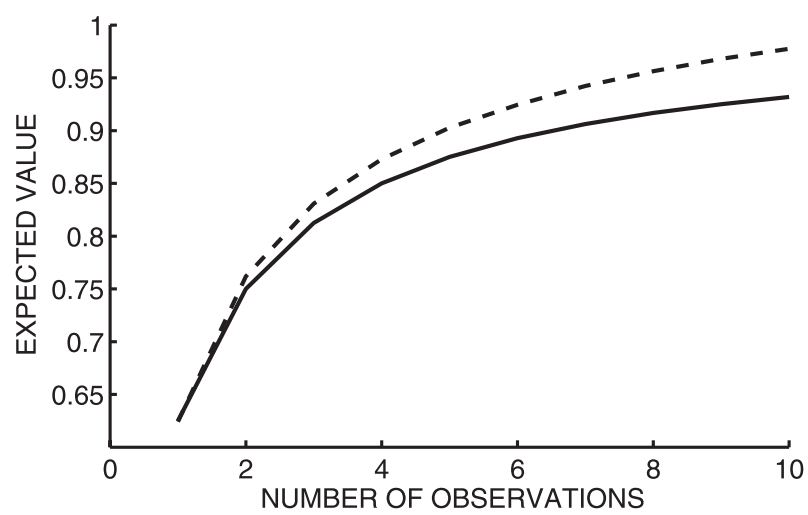

FIG. 2. Expected value of maximum observed wind speed vs $n$ for $v_{\max }=1, v_{0}=0.25$, and $\sigma=0$ (solid) and $\sigma=0.1$ (dashed).

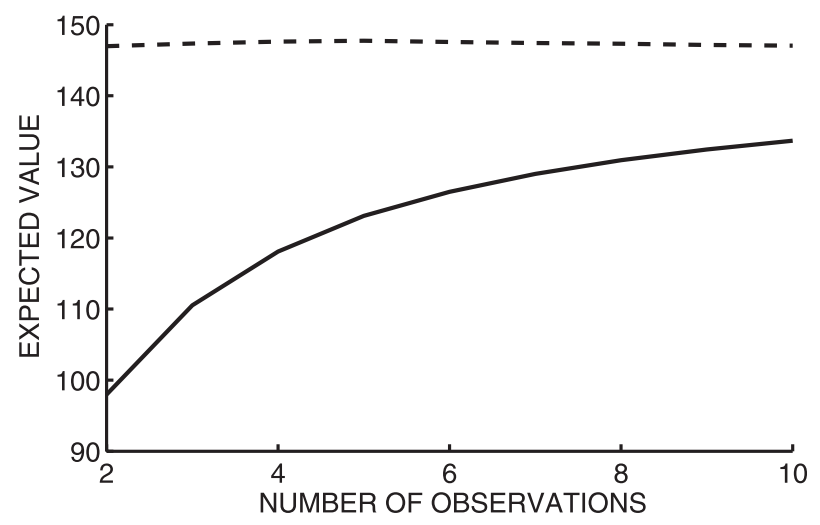

FIG. 3. Expected value of maximum observed wind speed (solid) and expected value of reconstructed maximum wind speed (dashed) vs $n$ for Hurricane Andrew.

39 6-hourly wind speed measurements covering a 228-h period with a maximum of $150 \mathrm{kt}$. These data were taken to represent the true evolution of maximum wind speed for Hurricane Andrew, with values between observation times reconstructed by linear interpolation. This profile is shown in Landsea et al. (2004b, their Fig. 4) and also in Emanuel (1999, his Fig. 2).

The simulation experiment proceeded in the following way. For each value of $n$ between 2 and 10, wind speeds were sampled at $n$ random times from this profile, and both the maximum $W_{(n)}$ of these $n$ wind speeds and the estimate $\hat{v}_{\max }$ based on it were recorded. The procedure was repeated a total of 10000 times. In Fig. 3, the averages of the 10000 values of $W_{(n)}$ and $\hat{v}_{\max } \operatorname{sim}$ ulated in this way are plotted against $n$. In this case, $\hat{v}_{\max }$ performed very well, on average, underestimating $v_{\max }$ by only around $3 \mathrm{kt}$ for $n$ between 2 and 10. In contrast, on average, $W_{(n)}$ underestimated $v_{\max }$ by more than $50 \mathrm{kt}$ when $n=2$ and almost $20 \mathrm{kt}$ when $n=10$.

The experiment was repeated for a small number of other hurricanes listed in Table 1. Wind speed data for these hurricanes were available online at the HURDAT Internet site, and the corresponding wind speed profiles are shown in Emanuel (1999). Table 1 reports the value of $v_{\max }$ and the average values over 10000 simulated

TABLE 1. Values of $v_{\max }$ and averages of more than 10000 simulations of $W_{(n)}$ and $\hat{v}_{\text {max }}$ for $n=3$ for selected North Atlantic hurricanes. All wind speeds are reported in knots.

\begin{tabular}{lcrcc}
\hline Name & Year & $v_{\max }$ & $\operatorname{Avg} W_{(n)}$ & $\operatorname{Avg} \hat{v}_{\text {max }}$ \\
\hline Gloria & 1985 & 125 & 87.5 & 116.7 \\
Gilbert & 1988 & 160 & 123.9 & 165.2 \\
Dean & 1989 & 90 & 79.4 & 105.9 \\
Hugo & 1989 & 160 & 129.6 & 172.9 \\
Andrew & 1992 & 150 & 110.1 & 147.1 \\
Opal & 1995 & 130 & 87.5 & 116.7 \\
\hline
\end{tabular}


random wind speed samples of $W_{(n)}$ and $\hat{v}_{\max }$ for $n=3$. With the exception of Hurricane Dean, the absolute relative bias of $\hat{v}_{\text {max }}$ is less than $10 \%$, with $v_{\max }$ underestimated in half the cases and overestimated in the other half. In contrast, by necessity, $W_{(n)}$ always underestimates $v_{\max }$ with a relative underestimation bias of up to $32 \%$. It is only for Hurricane Dean that the absolute bias of $W_{(n)}$ is smaller than that of $\hat{v}_{\max }$. The reason is that the wind speed profile for this hurricane is far from the model in (7) and, in particular, exhibits a plateau just below its peak. In overall terms, given its extreme simplicity, $\hat{v}_{\max }$ appears to perform well at correcting the underestimation bias of $W_{(n)}$.

\section{Discussion}

The purpose of this note has been to outline and illustrate a statistical formalism for exploring the effect of sparse random sampling on the maximum observed wind speed in a hurricane. The model considered here is clearly stylized and can be extended in a number of ways. In particular, realism could be gained through an explicitly spatial model of hurricane wind fields and their sampling. In some situations, it may be reasonable to assume that observers seek to avoid the highest winds. This would have the effect of exacerbating underestimation bias. On the wind speed side, the model for $v(t)$ in (7) can be extended to include a random component, so that $v_{\max }$ is itself a random variable.
The focus here has been on maximum wind speed. In some situations, interest centers on a function of it. For example, the power dissipation index of Emanuel (2005) depends on the cube of maximum wind speed. The underestimation bias in estimating $v_{\max }^{3}$ by $W_{(n)}^{3}$ is worse than that in estimating $v_{\max }$ by $W_{(n)}$. For example, in the absence of measurement error, when $v_{0} / v_{\max }=0.25$, the relative bias in estimating $v_{\max }^{3}$ is 0.51 when $n=2$ and 0.30 when $n=5$. As reported earlier, the corresponding values in estimating $v_{\max }$ are 0.38 and 0.13 .

Acknowledgments. The comments of two reviewers are acknowledged with gratitude. Support for this work was provided by NOAA Grant NA17RJ1223.

\section{REFERENCES}

David, H. A., and H. N. Nagaraja, 2003: Order Statistics. 3rd ed. Wiley-Interscience, $458 \mathrm{pp}$.

Emanuel, K. A., 1999: Thermodynamic control of hurricane intensity. Nature, 401, 665-669.

2000: A statistical analysis of tropical cyclone intensity. Mon. Wea. Rev., 128, 1139-1152.

, 2005: Increasing destructiveness of tropical cyclones over the past 30 years. Nature, 326, 686-688.

Landsea, C. W., and Coauthors, 2004a: The Atlantic hurricane database reanalysis project: Documentation for the 1851-1910 alterations and additions to the HURDAT database. Hurricanes and Typhoons: Past, Present, and Future, R. J. Murnane and K.-B. Liu, Eds., Columbia University Press, 177-221. , and Coauthors, 2004b: A reanalysis of Hurricane Andrew's intensity. Bull. Amer. Meteor. Soc., 85, 1699-1712. 